\title{
Determination of the impulsive Dirac systems from a set of eigenvalues
}

\author{
Ran Zhang ${ }^{1}$ \\ ${ }^{1}$ Nanjing University of Science and Technology
}

May 5, 2020

\begin{abstract}
In this work, we consider the inverse spectral problem for the impulsive Dirac systems on $\$(0, \backslash p i) \$$ with the jump condition at the point $\$ \backslash$ frac $\{\backslash \mathrm{pi}\}\{2\} \$$. We conclude that the matrix potential $\$ \mathrm{Q}(\mathrm{x}) \$$ on the whole interval can be uniquely determined by a set of eigenvalues for two cases: (i) the matrix potential $\$ \mathrm{Q}(\mathrm{x}) \$$ is given on $\$ \backslash \operatorname{Big}(0, \backslash$ frac $\{(1+\backslash$ alpha $) \backslash$ pi $\}\{4\} \backslash \mathrm{Big}) \$$; (ii) the matrix potential $\$ \mathrm{Q}(\mathrm{x}) \$$ is given on $\$ \backslash \operatorname{Big}(\backslash \operatorname{frac}\{(1+\backslash$ alpha $) \backslash \mathrm{pi}\}\{4\}, \backslash \mathrm{pi} \backslash \mathrm{Big}) \$$, where $\$ 0<\backslash$ alpha $<1 \$$.
\end{abstract}

\section{Hosted file}

Determination of the impulsive Dirac systems from a set of eigenvalues.pdf available at https://authorea.com/users/302404/articles/432466-determination-of-the-impulsive-diracsystems-from-a-set-of-eigenvalues 